\title{
A fascia lata mitral valve based on the 'frustum' principle
}

\author{
R. T. BR OWNLEE ${ }^{1}$ and A. K. YATES \\ Guy's Hospital, London
}

\begin{abstract}
A newly designed strut-supported 'frustum' fascia lata valve has been described for replacement of the mitral valve. It offers the advantages of autologous tissue leaflets, ease of construction and insertion, central flow through an unobstructed mobile annulus, no ventricular outflow obstruction, and minimal intracardiac prosthetic material. Initial clinical trials which have been carried out suggest that the action of this valve allows a close approximation of the haemodynamics and movement of the normal mitral valve.
\end{abstract}

Despite the refinement and standardization of cardiopulmonary bypass techniques, early mortality rates for mitral valve replacement remain between 10 and $20 \%$. In addition, the incidence of the late complications of thromboembolism and prosthesis malfunction remains high with one large cumulative series quoting a total one-year mortality rate for mitral valve replacements as $\mathbf{2 9 . 4} \%$ and a five-year mortality rate approaching $50 \%$ (Brewer, 1969).

Virtually all present mitral valve replacements show a stenotic pattern when studied by ultrasound techniques (Wharton, 1970). This finding is confirmed by postoperative haemodynamic studies of currently available prosthetic valves, which have revealed serious residual obstruction to flow at mitral valve level (Kloster, Herr, Starr, and Griswold, 1969 ; Linhart et al., 1969).

The object of the present work was to design a mitral valve replacement which closely approximated normal mitral valve mechanics and haemodynamics in an attempt to produce a more durable and physiological valve. Autologous fascia lata was chosen for valve construction because of the mounting evidence that autologous biological materials appear to offer the best chance of longterm satisfactory function (Senning, 1967; Ionescu et al., 1970).

Anatomico-physiological studies of the normal mitral valve have shown that the normal mitral annulus changes its shape from an oval one in diastole to a reniform one in systole, and that great horizontal mobility of the mitral annulus

Present address: University of Alberta Medical Center, Edmonton, Alberta, Canada. plays a considerable role in its normal function (Puff, 1968). Frater and Ellis (1961) have shown that the mitral annulus is not flat but oblique in the antero-posterior direction. It would, therefore, seem desirable to eliminate the wide, round, flat, and rigid sewing ring integral to all presently used mitral valve replacements. In addition, if the valve leaflets could be sewn directly to the mitral annulus, $1 \mathrm{~cm}$ or more effective flow orifice diameter would be gained over those valve replacement techniques employing a rigid sewing ring. Furthermore, a bicuspid valve whose cusp tip orifice diameter exceeds that of the inlet diameter would eliminate stenotic turbulent flow during diastole (Reid, 1970). Increasing the depth of the anterior leaflet would not only eliminate left ventricular outflow obstruction but allow for the normal obliquity of the mitral annulus and produce a 'normal' curved mitral cusp closure. In the case of the presently described mitral valve, prevention of prolapse of the fascia lata valve leaflets into the left atrium depends upon 'three point fixation' (two intraventricular strut projections and the mitral annulus itself). Mobility of the mitral annulus is accomplished by moving the 'horizontal' component of the valve support mechanism out of the annular region and into the posterior left atrium.

\section{TECHNIQUE OF VALVE CONSTRUCTION}

Before opening the chest, a sheet of fascia lata is removed through a longitudinal thigh incision. After trimming excess connective tissue, the fascia for the valve is cut out using a metal template (Fig. 1), the dimensions of which are based on those of normal 


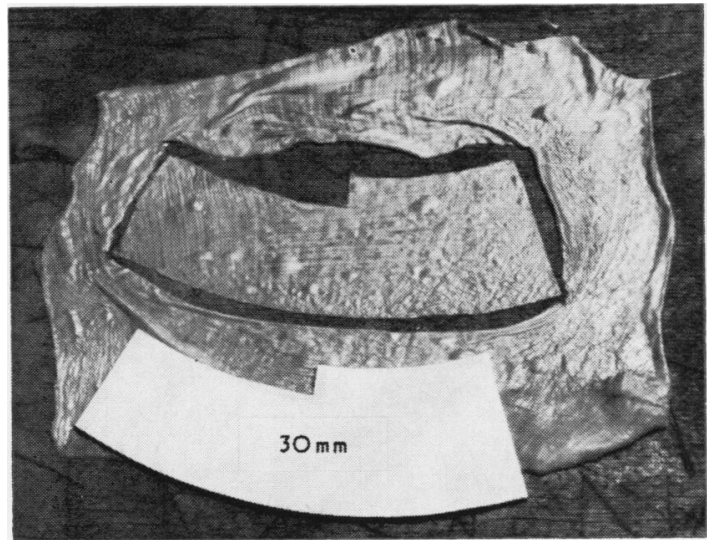

FIG. 1. After removal of attached connective tissue, the fascia is cut to a predetermined shape using a metal template.

mitral and aortic valves (Yates, 1971). A template for each 2-mm increment of mitral orifice size has been fabricated although the 28 to $32 \mathrm{~mm}$ size valves appear suitable for most adult mitral valve replacements. Selection of valve size can be made preoperatively, purely on the basis of estimation of heart size and the type of valve lesion found at cardiac catheterization. A $3 \mathrm{~mm}$ wide strip of thin Dacron is then attached to what will be the inside of the valve inlet orifice with $\overline{00000}$ Mersilene as reinforcement for the annular suture line (Fig. 2). What is to become the anterior mitral leaflet is somewhat longer than the posterior leaflet, allowing for the normal anteroposterior obliquity of the mitral anulus. Using continuous $\overline{000}$ Mersilene, the strip of fascia is converted into a truncated cone or frustum with the 'rough' side of the fascia and the Dacron reinforcing layer on the inside or inflow surface of the cone.

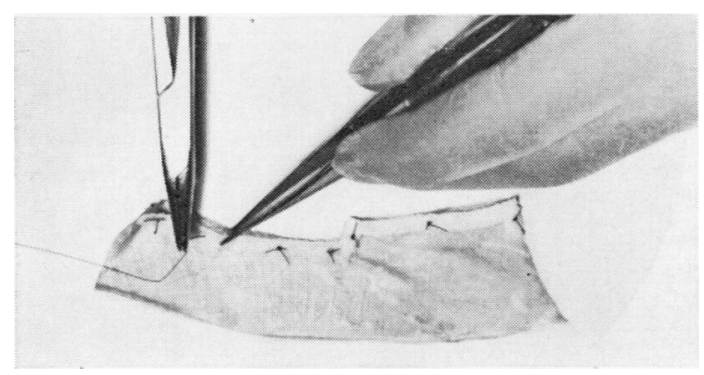

FIG. 2. A reinforcing layer of thin Dacron is attached to what will become the annular end of the mitral valve with interrupted horizontal mattress $\overline{00000}$ Mersilene sutures. The Dacron is placed slightly away from the fascial edge to facilitate subsequent suture into the mitral annulus.

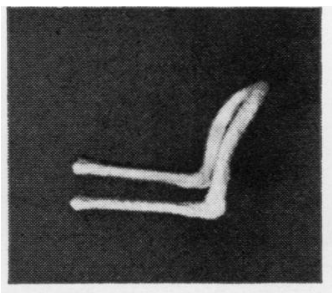

(a)

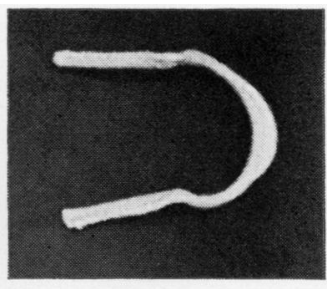

(b)
FIG. 3. Mitral frustum valve strut: (a) lateral view ; (b) antero-posterior view. For description, see text.

The supporting strut for the mitral 'frustum' valve is made of titanium covered with Dacron velour (Fig. $3)^{1}$ and consists of an intraventricular and a supraannular portion. The intraventricular 'prongs' are divergent from each other at their tips. The fascia is draped over the strut and attached to the Dacron sewing ridges with $\overline{000}$ Mersilene (Fig. 4). This suture line is terminated to within about $5 \mathrm{~mm}$ of the strut angle in order to facilitate subsequent suture of the fascia to the annulus behind the strut.

A geometric relationship between annulus size, depth of valve (i.e., length of prongs), and the degree of inclination of the valve lateral walls upon each other exists, which remains constant. This relationship, which is basic to the design of both frustum mitral and aortic valves, has been previously described by one of us (Yates, 1971). The supra-annular or 'hoop' portion of the strut is designed to maintain this geometric relationship of the intraventricular portion of the strut without in any way obstructing or fixing the mitral annulus, and lies in the posterior lateral left atrium rather than being sutured into the mitral annulus. Construction is completed in about 20 minutes, by which time the chest will have been prepared by an assistant, and no time has been lost in starting cardiopulmonary bypass.

\section{TECHNIQUE OF VALVE INSERTION}

Using graduated obturators, only enough mitral valve is excised to produce the desired orifice size (i.e., the diameter of frustum valve constructed). The frustum valve is then inserted into the mitral annulus, taking care to orient the long anterior leaflet directly beneath the left ventricular outflow tract. The fascia is then sewn directly to the mitral annulus with a continuous $\overline{00}$ Mersilene suture through the previously placed Dacron reinforcing strip. Because the suture line attaching the fascia to the strut has not been carried all the way to the top of the intraventricular portion of the strut, the strut may be easily moved aside to facilitate suturing of the fascia to the annulus behind it.

1Hypodermic Services, 1 Headlands Road, Liversedge. Yorks 


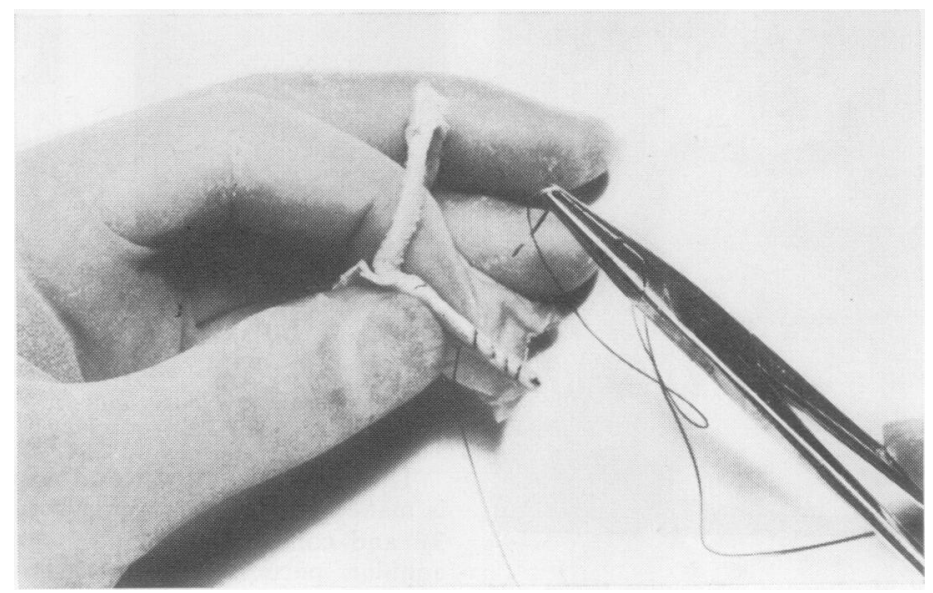

FIG. 4. Attachment of the fascia lata to the strut.

The annular suture line and valve competency can then be tested by allowing the left ventricle to fill with blood. Any paravalvular leaks may be closed at this time with interrupted sutures. The

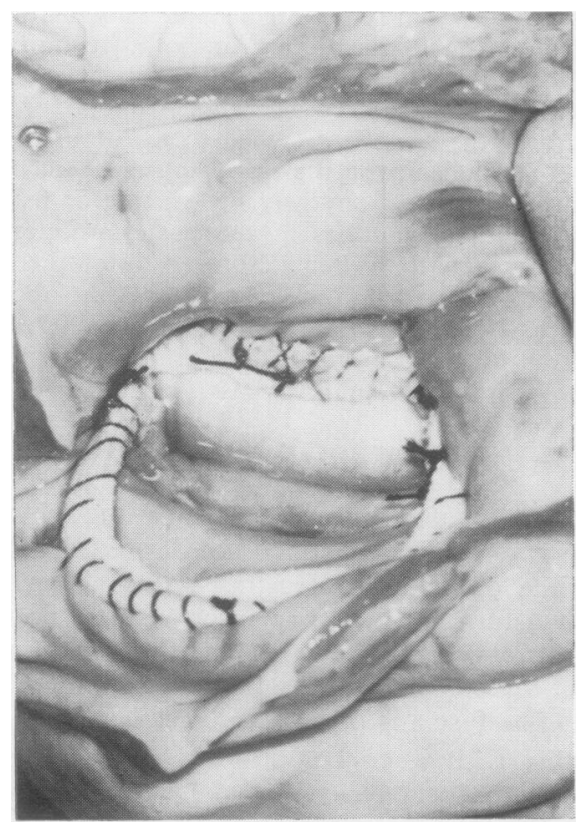

FIG. 5. Mitral frustum valve inserted in human cadaver heart viewed from the left atrium. The fascia lata cusps are fixed to the intraventricular portions of the strut, and directly to the mitral annulus. The 'supra-annular hoop' portion of the strut is fixed to the posterior left atrial wall. Left ventricular static pressure load to $300 \mathrm{mmHg}$. Note the 'normal' curved closure of the cusps. strut is then fixed to the annulus, fascia, and reinforcing Dacron with four interrupted $\overline{00}$ sutures and the supra-annular strut ring is fixed to the posterior left atrial wall by continuous $\overline{000}$ sutures through the Dacron sewing flange provided.

\section{PRE-CLINICAL TRIALS}

Valves of orifice sizes 14 to $40 \mathrm{~mm}$ were inserted into human cadaver hearts and tested repeatedly

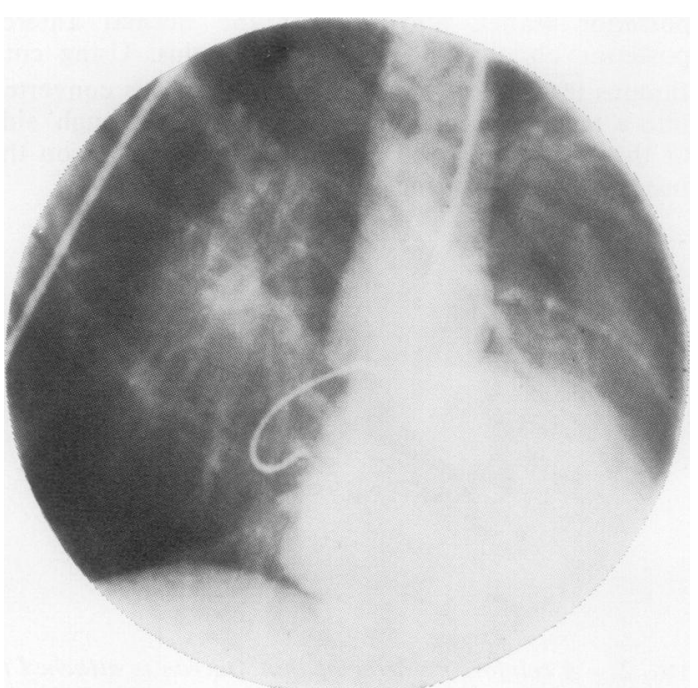

FIG. 6. Left ventricular angiogram in the right anterior oblique position five months after mitral valve replacement with a fascia lata frustum valve. No mitral regurgitation was observed. 
under left ventricular static pressure loads to in excess of $300 \mathrm{mmHg}$. No incompetence was observed. It is of some interest that frustum valve closure closely resembles that of the normal mitral valve (Fig. 5). Also, when the valve struts are correctly orientated, as described above, exposure of the left ventricular outflow through the aortic root reveals no portion of the mitral valve mechanism visible, suggesting that the left ventricular outflow obstruction will not represent a problem with this type of mitral valve replacement.

\section{CLINICAL TRIALS}

The above described bicuspid frustum mitral valve has now been inserted in 11 patients, the longest follow-up period being seven months, and early resuits are encouraging (Fig. 6). Immediate postoperative studies show no pressure gradients across the valve and normal left atrial pressure tracings. Preliminary ultrasound studies of cusp movement show a very wide amplitude of cusp movement and closure of the valve before the onset of ventricular systole, suggesting that the normal vortex flow pattern in the left ventricle during systole has been preserved (Reid, 1970).
The authors wish to express their thanks to Miss V. Wild for technical assistance.

\section{REFERENCES}

Brewer, L. A., III (1969). Statistical survey of 3,620 cases of heart valve replacement. Prosthetic Heart Valves, edited by L. A. Brewer, III. pp. 838-845. Thomas, Springfield Illinois.

Frater, R. W. M., and Ellis, F. H., Jr. (1961). The anatomy of the canine mitral valve. J. surg. Res., 1, 171.

Ionescu, M. I., Ross, D. N., Deac, R., Grimshaw, B. A., Taylor, S. H., Whitaker, W., and Wooler, G. H. (1970). Autologous fascia lata for heart valve replacement. Thorax, 25, 46.

Kloster, F. E., Herr, R. H., Starr, A., and Griswold, H. E. (1969). Hemodynamic evaluation of a cloth-covered Starr-Edwards valve prosthesis. Circulation, 39 and 40, Suppl. 1, 119.

Linhart, J. W., Barold, S. S., Hildner, F. J., Samet, P., Piccinini, J. C., Marsten, J. L., and Greenberg, J. J. (1969). Clinical and hemodynamic findings following replacement of the mitral valve with a Beall valve prosthesis. Circulation, 39 and 40, Suppl. 1, 127.

Puff, A. (1968). Zur funktionellen Morphologie des Mitralklappenapparates. Thoraxchirurgie, 16, 555.

Reid, K. G. (1970). Design criteria for a prosthetic orthotopic heart and mitral valve. Guy's Hosp. Rep., 119, 209.

Senning, A. (1967). Fascia lata replacement of aortic valves. J. thorac. cardiovasc. Surg., 54, 465.

Wharton, C. (1970). Personal communication.

Yates, A. K. (1971). A fascial frustum valve for aortic valve replacement. Thorax, 26, 184. 